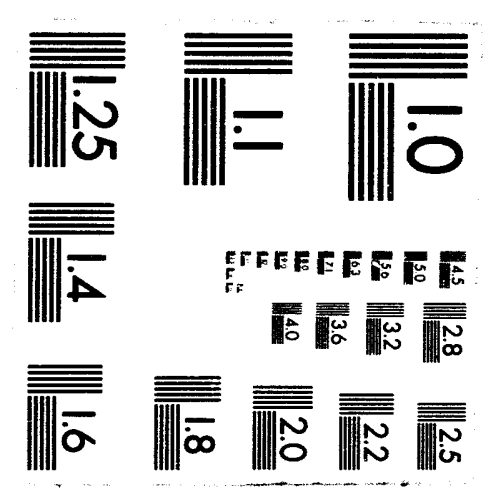



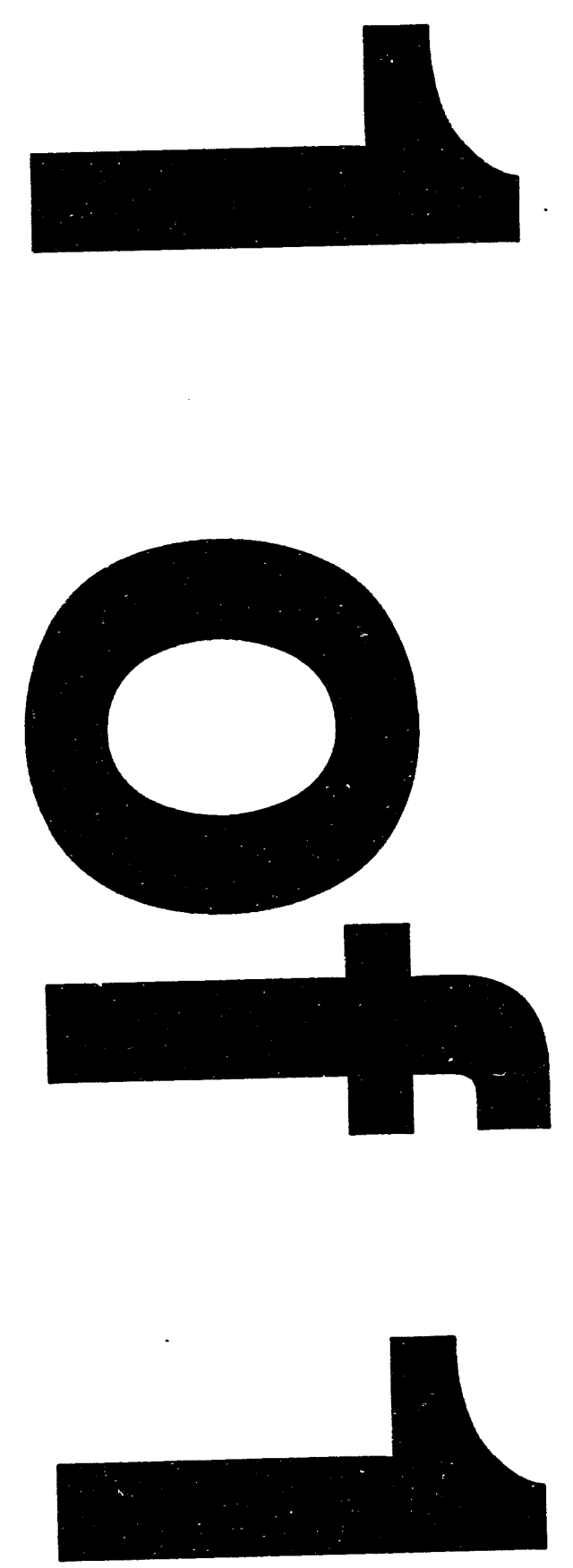
This report was prepared as an account of work sponsored by an agency of the United States Government. Neither the United States Government nor any agency thereof, nor any of their employees, makes any warranty, express or implied, or assumes any legal liability or responsibility for the accuracy, completeness, or usefulness of any information, apparatus, product, or process disclosed, or represents that its use would not infringe privately owned rights. Reference herein to any specific commercial product, process, or service by trade name, trademark, manufacturer, or otherwise does not necessarily constitute or imply its endorsement, recommendation, or favoring by the United States Government or any agency thereof. The views and opinions of authors expressed herein do not necessarily state or reflect those of the United States Government or any agency thereof.

\title{
UNIVERSAL SU(2/1) AND THE HIGGS AND FERMION MASSES
}

\author{
Yuval Ne'emant* \\ Raymond and Beverley Sackler Faculty of Exact Sciences \\ Tel-Aviv University, Tel-Aviv, Israel 69978 \\ cWolfson Chair Extraordinary in Theoretical Physics (TAU) \\ *Supported in part by the US DOE Grant DE-FG05-85ER40200; \\ also on leave from Center for Particle Physics, University \\ of Texas, Austin.
}

This work is dedicated to Larry Biedenharn, whose own key contributions to group theory, to its applicability and to the physical implementations have spanned the entire field.

\begin{abstract}
We review the SU(2/1) internal supersymmetry suggested by $D$. Fairlie and the author in 1979. The initial apparent difficuities were resolved when, with J. Thierry-Mieg, we understood that the gauging of a supergroup implies taking the usual Yang-Mills-like Principal (Double) Fibre Bundle as a "scaffold" and using its Grassmann algebra as parameter manifold for the supergauge. SU(2/1) Universality fixes the masses of the Higgs scalar field and the "top" quark around 100-200 GeV, in the same region as the $W$ and $Z$ masses. A "unified" supergauge, enclosing SU(3)colour $X$ SU(2) $\times U(1)$, predicts a fourth lepton generation in which the neutrino mass is of the same order.
\end{abstract}

\section{BACKGROUND: PARTICLE PHYSICS AND MATHEMATICS 1919-1992}

The progress of physics is often linked with mathematical advances we have been aware of this fact ever since the invention of the calculus. In our present paradigm for the fundamental physics of particles and fields, the first installment, namely QED, required the following physical tools: 1) F. London's (1927) reinterpretation of the electric charge and current as the Noether-derived conserved quantities corresponding to invariance under transformations of the quantum phase; 2) To complete the key concept of local gauge invariance, one also needed $H$. Weyl's two-step advance - first (1919) in gauging scale transformations (the group R) and then, after London's clariffcation, gauging the locally dependent group U(1). Mathematically, this was the birth of the Fibre Bundle concept [1]. 3) The development of Relativistic Quantum Field Theory (RQFT) by Tomonaga, Feynman, Schwinger and Dyson (1946-48); mathematically, the 
path-integral and related functional methods were essential tools. As a matter of fact these concepts have not yet fully matured mathematically and relevant studies are continuing.

The "Standard Model" which, at this time, represents the envelope of our paradigmal understanding, required in addition, 4) The quark model, whose discovery [2] stermed from the identification of the overall pattern of hadron states - namely "flavour"-SU(3) symmetry [3]. This evolution of symmetries relating to "internal" degrees of freedom represents a generalization of London's $U(1)$ of electric charge. On the mathematical front, this step ushered in a generalized use of semi-simple Lie groups. Particle physicists thereby followed in che footsteps of Weyl, Wigner, Racah, Elliott, Biedenharn and Moshinskj' - the prophets of the "group pest" [4] in Molecular, Atomic and Nuclear Physics. 5) as in the case of Weyl's $U(1)$, the local gauge version of SU(3) also involved two steps. In the first [3], the "flavour" group was locally gauged. It is, however, now regarded as a global symmetry, even though it does happen to involve a "universally"-coupled Yang-Mills multiplet of (massive) vectormesons, such as the rho, omega, etc. These are now relegated to the anonymity of "ordinary" quark-antiquark mesonic bound states, with their universal couplings reinterpreted as "phenomenological" features. In the second step, the "colour"-SU(3) group [5] was gauged, ylelding Quantum Chromodynamics (QCD). For both, the generalization of Weyl's "elch invarianz" to semi-simple Lie groups by C.N. Yang and R. Mills and independently by R. Shaw, was the crucial step. When the geometric nature of these theories became clear to physicists in the seventies, algebraic topology was a great help (instantons, monopoles, etc.). Physics, however, repaid its debt very rapidly and with largesse - gauge theories enabled Donildson and Freedman to make great advances in the topology of 3- and 4- dimensional manifolds. 6) for SU(2) left $\times U(1)$, Nambu, Goldstone, Weinberg, Higgs and others had first to develop the physical understanding of spontaneous symmetry breaking (SSB). It seems there was no need to invoke new mathematics.

The completion of the Standard model was followed by the emergence of two continuation programs: 7) Gauge-Unification, an embedding of SU(3) colour $\times$ SU(2) left $\times U(1) \subset G$ in a simple Lie group $G$, with the aim of reaching with respect to the break $G \rightarrow S U(3) \times[S U(2) \times U(1)]$, a level of understanding comparable to that of the SSB of $\{S U(2) \times U(1)\} \rightarrow U(1)$ elect. . This program did not involve new concepts mathematically at first, but it encountered the "hierarchy" problem, 1.e. difficulties in the preservation of two distinct SSB systems with a large energy-level separation. Spacetime supersymmetry [6], emerging independently of some earlier mathematical beginnings, provides one possible answer, whose validation requires the discovery of a rich spectrum of complementary states in the region of .5 - $2 \mathrm{TeV}$. 8) Quantum Gravity, 1.e. the quantization of a gauge theory which does not follow the Yang-Mills pattern. Here too, supergroups and supergeometry were moblilized, yielding Supergravity and Superstrings. These theories manage to involve Unification structures as well, in the aboye sense.

In 1992, we do not yet know whether supergroups and supermanifolds will indeed provide the physical answers in these programs. In mathematics, the physics interest has brought about the classification of the simple Lle superaigebras [7], completed by $V$. Kac and about important advances in supergeometry by Kostant, Berezin, Leites, De Witt, Bachelor and Rogers [8].

\section{GEOMETRICAL DESCRIPTION FOR GHOSTS AND BRST}

In 1976-77, I was mostly Involved in trying to understand the geometry of gravity - and of the newly born supergravity - gauge theories which were gauging spacetime symmetries. After starting an iterative 
gauge approach in superspace with the Caltech group [9], I collaborated with T. Regge [10] in developing a geometric gauge approach with Lie derivatives and the non-Lie algebras they generate, with structure functions instead of structure constants. The geometry involved a "softened" version (in the sense of Salvator Dali's "Soft Self-Portrait" or of his watches..) of the relevant Group Manifold, with spontaneous fibration: Spacetime, the maniflold of the translations subgroup, becoming a base manifold in an effective bundle - by the equations of motion, with the Lorentz group as fibre. For supergravity, this happens to 8-dimensional superspace, and the "local supersymmetry" transformations are generated by the relevant spinorial super-Lie derivative.

I spent some time in 1977 in Paris and lectured about this work. My audience included a graduate student, J. Thierry-Mieg, who got interested and participated in the completion of this program [ii]. By the time I returned to Paris in 1978, Jean had applied our technique of working in the full dimensionality - a must for the Soft Group Manifold (SGM) - to Principal Fibre Bundles as well. This seemed redundant, previous work having either been done globally and invariantly, using the exterior calculus, or having been limited to the horizontal projection of a selected section, i.e. to the gauge potential field $A^{a}{ }_{\mu} d{ }^{\mu}$. Picking a section and working nevertheless in the full dimensionality, brought out the seemingly uninteresting components of the connection. In the following, $\mathrm{y}^{\mathrm{m}}$ is the coordinate of $G$, the gauged group; for reasons that we shall explain later, we have doubled the gauge group from $G$ to $G \times G$ in these formulae.

$$
C=\left[A^{a} \mu d x^{\mu}+x^{a} m d y^{m}+x^{\star a} \underline{m} d y \star m\right]\left\{\lambda_{a}\right\}
$$

$\lambda_{a}$ is the Lie algebra matrix basis. Jean suggested that $x^{a}=x^{a} m d y^{m}$ might represent the ghost field, first introduced by Feynman, to preserve off-mass-shell unitarity, further developed by De Witt and by Faddeev and Popov. As a 1-form it anticommutes, its Lorentz scalar nature not-

withstanding. Moreover, we soon found [12] that expanding similarly the full-dimensionality exterior derivative,

$$
\Delta=d+s+s^{*}, d=d x \mu \partial_{\mu}, s=d y^{m} \partial_{m}, s^{\star}=d y * \underline{m} \partial_{\underline{m}},
$$

yields the BRST unitarity equations [13], which are nothing more than the Maurer-Cartan structure equations of the bundle, guaranteeing that the curvature 2-form be purely horizontal, 1.e. making $\mathrm{dx} \wedge d y$ and dyAdy com-

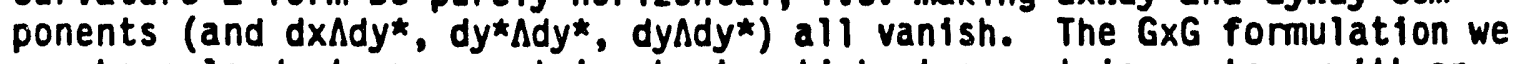
use here leads to a symetric ghost-antighost geometric system, with an appropriately symmetric BRST/antiBRST algebra, first constructed by Curci and Ferrari [14] (in the original BRST formulation, the anti-ghost transformation did not lend itself to geometrization). The geometrical identification of the ghosts and of the BRST operator and equations was criticized at first, but is accepted and used everywhere now, including the former critics, except that for some unexplained reason, Stora designates it now as "the Russian formula".

\section{EMERGENCE OF SU(2/1)}

This is how, sometime early in 1979, I noted that, should we be gauging a supergroup, the ghosts of the odd plece of the superalgebravalued connection would be plain bosonic flelds. Moreover, should the supergroup be SU(2/1), they would have isospin-hypercharge quantum numbers similar to those of the $K$-mesons in an SU(3) octer; but these are precisely the quantum numbers assigned to the Higgs field in SU(2) $x$ $U(1)$. In the same "ghost" multiplet (the vertical part of the SU(2/1) connection) the other four fields would be nreciselv the canonical ohost.s 
of the ( $(W F, Z, Y)$ vector-mesons. Looking at the leptons, their SU(2) $X$ $U(1)$ assignments fitted perfectly with the defining 3-dim. representation of SU(2/1). I did not yet know what to do with the quarks, and sald so in the paper [15]. The Weinberg angle came out as $\sin ^{2} \theta=.25$, a value close to the experimental .22 - .23 (which it might reproduces, with renormalization corrections, from $100 \mathrm{GeV}$ downwards; however, this is based on normalizing the algebra's matrices $\mu_{a}$ by

$$
\operatorname{tr}\left(\mu_{a} \mu_{b}\right)=2 \delta_{a b}
$$

which befits su(3) rather than su(2/1), at first sight at least. However, the supertrace, which would have been the obvious choice, is useless: All it does is define a metric which is then used to define upper-index matrices

$$
\operatorname{str}\left(\mu_{a} \mu_{b}\right)=2 g_{a b}, \mu_{a}=g_{a b} \mu^{b}
$$

This was one of the paradoxical results, at first.

David Fairlie conceived the same idea of the SU(2/1) supergroup [16], with a different motivation, related to the method of dimensional reduction, popular at the time. His Higgs fields were the residual postreduction components of "normal" Yang-Mills fields originally. The result was the same. Following our proposal, about ten papers were written by various authors (Jarvis, Taylor, etc.), applying SU(2/1), but this stopped after a while. The novelty of applying a supergroup as an internal symmetry gauge meant that, in the beginning, there were many problems of interpretation and apparent paradoxes, which gave the theory a speculative tinge. One obvious issue, the quarks, was quickly solved since Nahm, Scheunert and Rittenberg had studied [17] the representations of sl $(2 / 1)$ and shown that besides the 3 , there was a fundamental 4 , related to the homomorphism $S U(2 / 1) \sim \operatorname{OSp}\left(\frac{2}{2} / 2\right)$. This is the ortho-symplectic supergroup with a metric $\left\{10^{2} \times 12\right\}$ : The even subgroups su(2) $\sim \operatorname{sp}(2)$ and $u(1) \sim 50(2))$. The 4 carries precisely the internal quantum numbers of the 4 quark chiral fièlds, up to addition of an overall constant. Moreover, if this constant is set so as to make electric charges become integers (e.g. adding -2/3), all matrix elements in the 4 th column and row of the representation matrices vanish and $4 \rightarrow \underline{3}, 1 . e$. the group "predicts that there are 4 fractional charge fièlds, but only 3 when for integer charges [18]. Note the economy in the number of (arbitrary) multiplet assignments: $2(I=1 / 2 \& I=0)$ for leptons and $3(I=0, I=1 / 2$, $I=0)$ for quarks, plus as many $Y_{w}$ assignments, in su(2)xu(1) (1.e. a total of 10) are replaced by 2 multiplets and a constant (total: 3 choices) in su(2/1)! For the mesons, of course, we remove the arbitrariness in the selection of the I and $Y_{W}$ assignments for the Higgs fleld - a reduction of 2. In toto, a reduction of 9 in the multiplet assignments.

Two mysteries were resolved in $1982[19,20]$ : (a) The above mentioned emergence of the SU(3) metric and normalization (in getting the Weinberg angle) and (b) new types of ghosts which appear in the gauge (adjoint) vector meson multiplet. Indeed, together with the (WF, $Z, V$ ) $J=1$ gauge particles of $S U(2) \times U(1)$, we now have four $K^{\star}-1$ ike vector mesons, but they anticommutel The resolution of this riddle required a new understanding of the way in which a supergroup is gauged (at least for an internal supersymmetry, but possibly always). This advance was achieved independently of simflar results, which were soon to be obtained in mathematics by Quilien [21]. In 1986, I showed [22] that the prediction for the miss of the Higgs field

$$
M_{h}=2 M_{w}
$$

could be derived even without gauging the supergroup, taking instead a minimalist approach, 1.e. assuming $S U(2 / 1)$ to be a phenomenoiogical glo- 
bal flavour-type low-energy symmetry. With Shlomo Sternberg in 1980, we developed the appropriate (phenomenological) "superunification" [23],

$$
\left[S U(2 / 1)_{w-e m} \times \operatorname{SU}(3)_{c o l}\right] \in \operatorname{SU}(n / 1)
$$

which predicts $2^{n-5}$ generations. There was need, for this purpose, to construct the representations of $s 1(n / 1)$. It turns out that $n=7$ has the advantage of being anomaly-free [24]. It predicts 4 generations; since the experimental bound for generations with massless or light neutrinos is 3, the 4 th generation should involve a neutrino with a mass higher than half the $Z$ mas, roughly.

In 1990, Sternberg and I returned to the gauging of the supergroup, with improvements exploiting the mathematical advances in the theory of the "superconnection" $[25,26]$. In 1991, with C.Y. Lee, we improved and completed the relevant geometricall-derived BRST for SU(2/1), guaranteeing unitarity. We also [27] checked the possibility of generating, the $-m^{2} \Phi^{2} h$ negative mass-term, needed to trigger the SSB, by using radiative corrections à la Coleman-Weinberg. This is the only term in the SSB mechanism which did not appear naturally from $S U(2 / 1)$, in our original treatment. The radiative corrections mechanism gives a low-mass Higgs and I do not favour this approach, as a result. An alternative derivation was suggested in 1991, based on the inclusion of a discrete operator in the overall SU(2/1) covariant derivative [28]. This idea was an innovation, in what amounted otherwise to a plain (twelve-years late) rediscovery of the whole theory, during 1990-91, by Coquereaux, Scheck, and others, spurred by independent developments in non-commutative geometry, by $A$. Connes and $h$ is group [29]. Returning to the couplings, SU(2) $x$ $U(1)$ introduces $g$ and $g$ 'as gauge couplings (or $g$ and $\operatorname{tg} \theta$ ), $\lambda$ for the $\theta^{*} h$ potential and 2 different $c$ Yukawa couplings $c \Psi+\Phi_{h} \Psi$ for leptons and for quarks, aside from $-m^{2}{ }_{h}^{2}$ and $\left\langle 0\left|\omega_{h}\right| O\right\rangle=v$, which remain free in SU(2/1). The first 3 couplings are replaced by a single one. In addition, with the Higgs field as part of the gauge multiplets, its coupling to fermions should be universal - provided we disentangle a related complication, perhaps in the framework of SU(7/1), 1.e. which of the lepton + quark sets get their mass only from the Higgs field which is included in SU(2) left $X U(1)$ c SU(2/1) (not to be confused with another SU(2/1) subgroup of SU(7/1) which makes the generations), rather than from other pleces of the overall Higgs field of the unified SU(7/1). Since a universal coupling implies masses of the order of $v$, it seems the 3rd or 4th family fit best with this description phenomenologically.

Note that the correlation between gradings and chirality assignments is no surprise [30], it is built in the chiral structure of Weak Interactions. The fact that the lepton and quark multiplets' $S U(2) \times U(1)$ quantum numbers correspond to supermatrices with $\operatorname{str}(Q)=0$ is due to the observation that electrically charged particles have mass (from renomal1zation?) and thus there is always a right-fermion for each charged leftfermion. Since SU(2) means $\operatorname{tr}\left(I_{W}\right)=0$ and there is no right-I $I_{W}$ implies $\operatorname{str}\left(I_{W}\right)=0$, and by the analog of the Gell-Mann Nishijima formula, $\operatorname{str}\left(Y_{W}\right)=0$. Thus $S U(2 / 1)$ just represents a "natural" embedding within the smallest supergroup with this supertracelessness.

There is no true spin-statistics problem for the lepton or quark multiplets. The odd generators contain Parity (the $\gamma^{0}$ matrix), since they relate left and right chiralities. They operate on $(1 / 2,0)+(0,1 / 2)$, behaving as $(1 / 2,1 / 2)$. Acting on the $S L(2, C)$ representation, they connect $1 / 2$ to $O$ (and similarly in $S L(2, C)_{R}$ ). The $R$ state is thus a spinless "boson" under $S L(2, C)_{L}$ and vice versa. We use doubled representations [18]. Note, however, that only the left-SU(2/1) is gauged. 
G is a supergroup gauged internally, $\Gamma$ its Lie superalgebra $\Gamma=\Gamma^{+}+\Gamma^{-}, \Gamma^{+}$the even and $\Gamma^{-}$the odd generators

$$
\begin{aligned}
& {\left[\Gamma_{\hat{g}}, \Gamma_{b}\right]=i f_{\hat{a}} \hat{c} \Gamma_{\hat{c}} \text {. }} \\
& {\left[\Gamma_{\hat{a}}, \Gamma_{\mathfrak{q}}\right]=1 \mathrm{faq}_{\mathfrak{q}} \Gamma_{\mathfrak{j}} \text {, }} \\
& \left\{\Gamma_{\mathfrak{l}}, \Gamma_{\mathfrak{j}}\right\}=d_{\mathfrak{q}} \hat{\mathfrak{I}} \Gamma_{\hat{\mathfrak{a}}} \text {, } \\
& \text { with } \Gamma_{\mathfrak{a}, \mathfrak{b}} \in \Gamma^{+} \text {and } \Gamma_{\mathfrak{q}, \mathfrak{j}} \in \Gamma^{-} \text {. }
\end{aligned}
$$

Selecting a section over the superbundle, $x$ is the (spacetime) horizontal coordinate, $y$ the (internal) vertical. We construct the Grassmann algebra $\Lambda(x, y)$ over both, with $\Lambda=\Lambda^{+}+\Lambda^{-}$the even and opd pieces $\left(\Lambda^{0}, \Lambda^{2}, \ldots\right)=\Lambda^{+},\left(\Lambda^{2}, \Lambda^{3}, \ldots\right)=\Lambda^{-}$; for forms in $x, \Lambda_{1}$ : pieces $n$, whereas $n^{\prime} S^{\prime} N,{ }^{\prime} N=\operatorname{dim}^{\prime} \Lambda^{2}(y)$ 'for forms in $y$. Notice that the $y$ coordinate itself is commutative, like $x$; to supply an anticomuting parameter for $\Gamma_{1}$ we simply use odd order forms, e.g.

$$
\Gamma_{\mathfrak{q}} \cdot f^{i_{m}}(x, y) d y^{m}=\Gamma_{\mathfrak{q}} \cdot F^{i} \text {. }
$$

The anticommutativity property of the parameter $F^{1}$ now derives from its being a one-form in the $y$ variable. The carets over the indices indicate the pressence of a superalgebra, 1.e. the caret over the 1 index contains the information about the anticommutation property, which is not yet present in the function $f^{i}(x, y)$. Here the 1 index stands for the same subalgebraic quantum numbers, but taken within the related (even) Lie algebra within the "Hermitian Lie algebra" [31]. Such a Hermitian Lie algebra occurs when the same basic set of generators can close either as a Lie algebra, or, by selecting a subalgebra $\Gamma^{+}$and imposing a $Z(2)$ grading and anticommutation rules for $\Gamma^{-}=\Gamma / \Gamma^{+}$, they close on a superalgebra.

For our application, we use $U(3)-U(2 / 1)$. For the i-1 transition, the transformation relates only to the change in the generalized Lie bracket, with $j-j$, from $\{1, j\}$ to $[1, j]$; for the g-a transition, the change is only in the decomposition gver the $\left(\mu^{8}, \mu^{0}\right)$ versus $\left(\lambda^{0}, \lambda^{0}\right)$ vectors in the $(8,0)$ plape, fince $s \operatorname{tr}\left(\mu^{8}\right)=0$, but $\operatorname{tr}\left(\lambda^{8}\right)=0$.

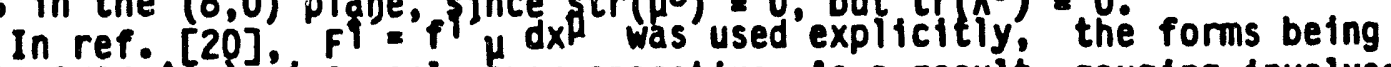
taken over $A(x)$, 1.e. only bver spacet ime. As a result, gauging involved higher tensors as gauge fields (such as the Kalb-Ramond B ${ }^{\prime}$ ). However, we shall now show that the identification of the Higgs field used $\Lambda(y)$, an important point in the understanding of the emergence of the $U(3)$ metric.

Connections C over a Principal Fibre Bundle (= a Yang-Mills gauge theory) enter $D=\Delta+C$ and acting on forms (coordinates $x, y$ ) increase their degree by one, with $D=D++D \rightarrow$ (vertical and horizontal - projecting over a selected section) $\Delta=d+s$ as in $(2.2)$ and $C=A+X$ as in (2.1).

Changing now to a supergroup, but staying still with the Yang-Mills picture in which $y$ (and not yet $A$ ) spans the fibre, we can immediately identify the Higgs field. The conne tion $C$ is still an anticormutative one-form and so are $\underline{A}$ and $X$. However,

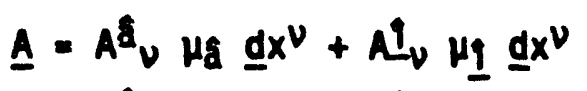

$$
\begin{aligned}
& X=x^{a_{m}} \mu_{g} d y^{m}+x_{m} \mu_{\underline{q}} d y^{m} .
\end{aligned}
$$

where we have underlined in each term the factors inducing fermion beha- 
viour, whether it is the odd degree of the form or the odd part in the superalgebra. We note that whereas $x^{a}=x^{a} m d y^{m}$ is indeed a fermionic scalar ghost field, $x^{i}=x_{-m} d y^{m}$ has boson statistics (the fermi feature in $X$ is supplied here by the $\vec{\mu}$ matrices in the algebra. This is how SU(2/1) had originally occured to me [15], as I related. Here the Higgs is a one-form, valued in the odd part of the superalgebra.

We now change to the "superconnection" geometry of refs. $[20,21,25$, 26]. The fibre is no more spanned by $y$, it is spanned by $\Lambda(y)$. The parameters

$$
F^{\hat{a}}(x, y) \in \Lambda^{+}(x, y), F^{\dagger}(x, y) \in \Lambda^{-}(x, y) .
$$

The gauge field has the quantum numbers of the parameters it gauges (this is the essence of the Yang-Mills idea) and a degree higher by one, in the variable of the relevant parallel-transport (covariant derivative) it spans. For $F^{a}(x, y)$ nothing has changed if it is taken from $\Lambda^{0}(x, y)$; should it, however, involve a higher even degree, such as $\Lambda^{2}(x, y)$, the relevant piece of the connection is the gauge totally antisymetric tensor $c^{a}$ uvo of [20]. Now take the action of the odd generator $\Gamma_{9}$, the $F \underline{\varepsilon}$ $\Lambda^{3}(x, y)$ parameters, one-forms in dym. For the vertical ("the gñost")

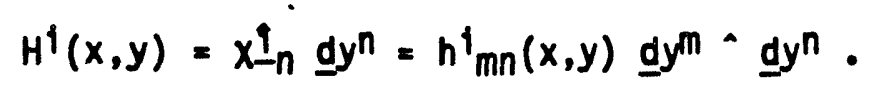

$H^{i}$ (our former $X^{f}$ ) is now an even-order form in dym [20]. The transition from the Principal Fibre Bundle (Yang-Mills) geometry, for an internal supergauge, to that of the Superconnection thus puts the $\phi_{h}$ field into the even part of the Grassmann algebra and with the relevant $U(3)$ index, instead of $U(2 / 1)$. As a matter of fact, we can generalize the result and directly identify ${ }^{1} h$ with a zero-form $[25,26]$. The supercurvature involves ( $\mathrm{da}^{\mathrm{a}} \mathrm{j}$ ), which is then squared, yielding the $\lambda \Phi^{\text {" term. }}$ We get the $\sin ^{2} \theta=.25$. However, $U(3)$ is not simple, and we should check whether the $\lambda$ coupling is preserved in the renormalization procedure.

\section{REFERENCES}

[1] C.N. Yang, in To Fulfill A Vision (Proc. Einstein Centen., Jerusalem (1979), Y. Ne'eman, ed., Addison Wesley Pub., Reading, Mass. (1981), pp. 3-11. See also C.N. Yang, Ann. NY Acad. Sc1., 294 (1977) 86.

[2] H. Goldberg and Y. Ne'eman, Nuo. Cim. 27 (1963) 1; M. Gell-Mann, Phys. Lett. 8 (1964) 214; G. Zweig, unpub.

[3] Y. Ne'éman, Nucl. Phys. 26 (1961) 222; M. Gell-Mann, Caltech report CTSL-20, unpub. and Phys. Rev. 125 (1962) 1067.

[4] H. Weyl, Raum, Zeit und Materie, Springer Verlag, Berlin (1920).

[5] M.Y. Han and Y. Nambu, Phys. Rev. 139B (1965) 1006; M. Gell-Mann, H. Fritzsch and H. Leutwyler, Phys. Lett. B47 (1973) 365; S. Weinberg, Phys. Rev. Lett. 31 (1973) 494.

[6] Y.A. Golfand and E.P. Likhtman, JETP Lett. 13 (1971) 452.

J. Wess and B. Zumino, Nucl. Phys. B70 (1974) 39.

[7] L. Corwin, Y. Ne'eman and S. Sternberg, Rev. Mod. Phys. 47 (1975) 573. P.G.0. Freund and I. Kaplansky, J. Math. Phys. 17 (1976) 228. V.G. Kac, Func. Anal. 9 (1975) 263.

[8] B.S. DeWitt, in Relativity, Groups and Topology, C. DeWitt and B.S. Dewitt eds., Gordon and Breach, NY (1964) 725; F.A. Berezin, The Method of Second Quantization, Academic Pr., NY (1966); B. Kostant, In Springer Verlag Lecture Notes in Math., 570, Berl in (1977), 177; M. Batchelor, Transa. Am. Math. Soc. 258 (1980) 257; A. Rogers, J. Math. Phys., 21 (1980) 1352.

[9] L. Brink et al., Phys. Lett. B76 (1978) 417.

[10] Y. Ne'eman and T. Regge, Riv. Nuo. Cim. (Series 3) 1, N5 (1978). 
[11] J. Thterry-Mieg and Y. Ne'eman, Ann. Phys (NY) 123 (1979) 247.

J. Thierry-Mieg, Thèse de D. d'Etat (U. IE Par1s Sud, Orsay, 1978);

J.Math.Phys. 21 (1980) 2834; Nuovo Cim. 56 (1980) 396: Y. Ne'eman,

T. Regge and J. Thierry-Mieg, Proc. 19th Int. Conf. High Energy Physics,

Tokyo, 1978, S. Homma et al. eds., Tokyo (1979), Pp. 552-554.

[13] C. Becch1, A. Rouet and R. Stora, Com. Phys., 42 (1975) 127.

[14] G. Curc1 and R.'Ferrar1, Nuo. Cim. A30 (975) 155.

[15] Y. Ne'eman, Phys. Lett. B81 (1979) 190.

[16] D.B. Falrlie, Phys. Lett. B82 (1979) 97.

[17] M. Scheunert, W. Nahm and V. Rittenberg, J. Math. Phys. 18 (1977) 155.

[18] $Y$. Ne'eman and J. Thierry-Mieg, Springer terlag Lect. Notes in Math. 836 (1980) Pp. 318-348 (Proc. Salamanca \$79) A. Perez-Rendon ed.; see also (same authors) Proc. Nat. Acad. Sci. USA 77 (1980) 720.

[19] J. Thierry-Mieg and Y. Ne'eman, Nuo. Cim. A71 (1982) 104.

[20] J. Thierry-Mieg, Y. Ne'eman, Proc. Nat. Sci. USA, 79 (1982) 7068.

[21] D. Quilien, Topology, 24 (1985) 89.

[22] Y. Ne'eman, Phys. Lett. B181 (1986) 308.

[23] Y. Ne'eman, S. Sternberg, Proc. Nat. Acac Sc1. USA 77 (1980) 3127.

[24] Y. Ne'eman and J. Thierry-Mieg, Phys. Le =. B108 (1982) 399.

[25] Y. Ne'eman, S. Sternberg, Proc. Nat. Acac Sci. USA 87 (1990) 7875.

[26] Y. Ne'eman, S. Sternberg, in Symplectic eometry and Mathematical

Physics (Proc. Intern. Conf. Diff. Geom. 'n Phys., honouring J.M.

Souriau, Marseflles, 1990), P. Donato et il. eds., Birkhauser, Boston (1991), 326.

[27] C.Y. Lee and Y. Ne'eman, Phys. Lett. B26. (1991) 389.

[28] R. Coquereaux et al, Nucl. Phys. B353 (151) 689; R. Haussing et al, Phys.Lett. B260 (1991) 125; R. Coquereaun Phys.Lett. B261 (1991) 449.

[29] A. Connes, Publ. Math. IHES 62 (1985) 41.

[30] $Y$. Ne'eman, in Unification of the Fundametal Particle Interactions,

S. Ferrara et al eds., Plenum Press, NY (.980), Pp. 89-100.

[31] S. Sternberg and J. Wolf, Trans. Amer. Man. Soc. 238 (1978) 1. 


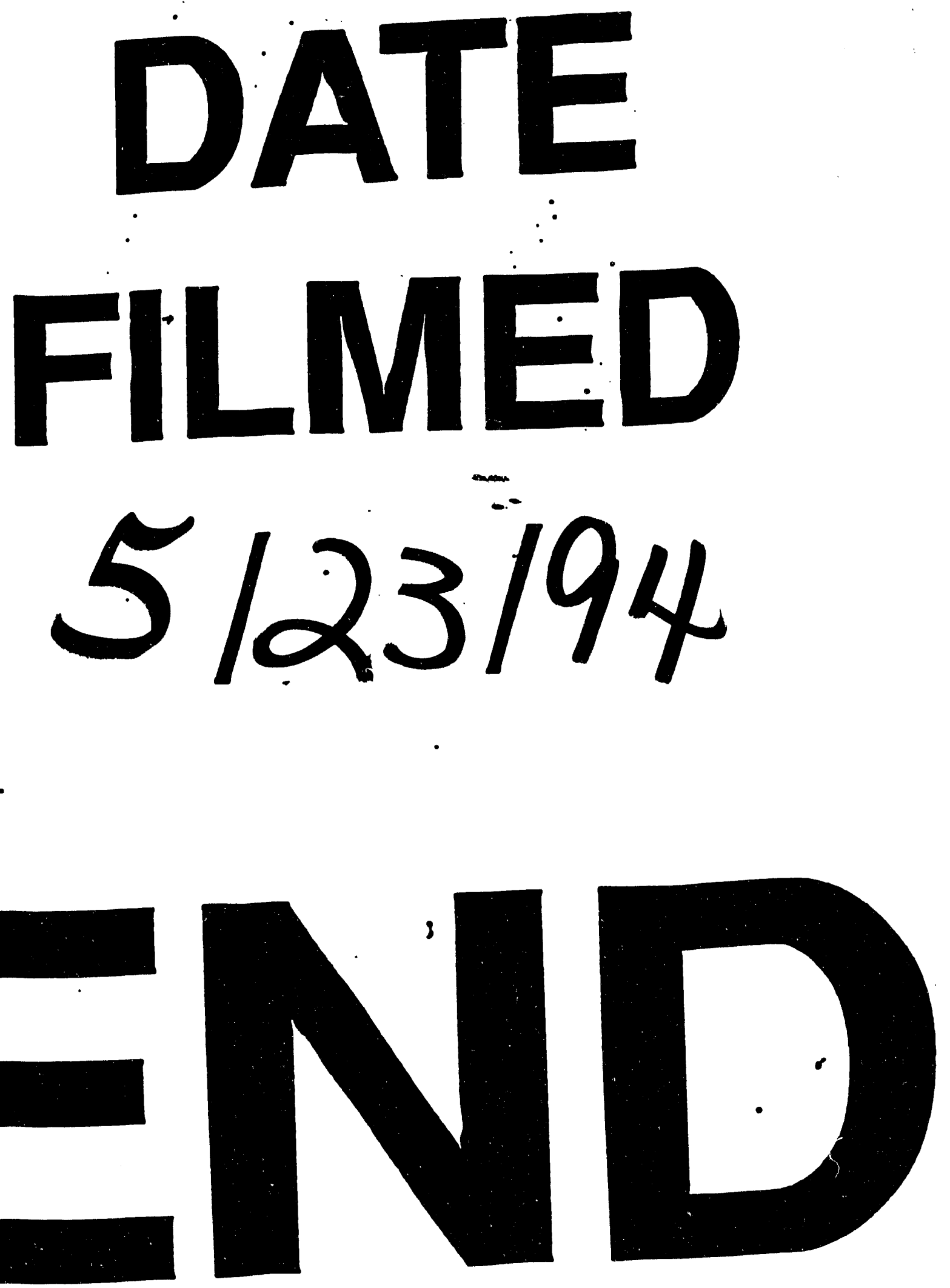




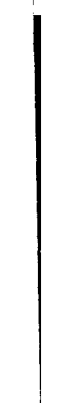

